\title{
Metabolic characteristics of acute necrotizing pancreatitis and chronic pancreatitis
}

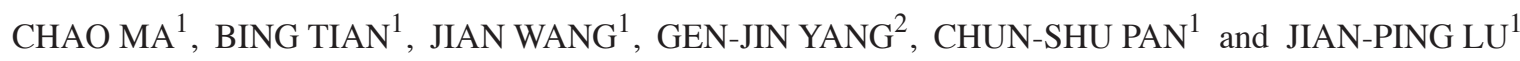 \\ ${ }^{1}$ Department of Radiology, Shanghai Changhai Hospital; ${ }^{2}$ Pharmaceutical Analysis and Testing Center, \\ School of Pharmacy, The Second Military Medical University, Shanghai 200433, P.R. China
}

Received January 10, 2012; Accepted April 10, 2012

DOI: $10.3892 / \mathrm{mmr} .2012 .881$

\begin{abstract}
The etiology and pathogenesis of pancreatitis remains unclear. In the presence of pancreatic inflammation, metabolite abnormalities appear before transformation of tissue structure and changes in functions occur. Detection of abnormalities in metabolite levels facilitates a greater understanding of the pathophysiological events and aids in the early diagnosis of the disease. In this study, metabolic profiles from the pancreas of Wistar rats were examined using high-resolution proton magic angle spinning nuclear magnetic resonance (MAS NMR) spectroscopy to investigate the metabolite indicator(s) of acute necrotizing pancreatitis (ANP) and chronic pancreatitis (CP). The animals were divided into three groups: those with histologically confirmed ANP $(n=7)$, those with CP $(n=6)$ and a control group $(n=9)$. The processed NMR spectra were analyzed using principal component analysis (PCA) to extract characteristic metabolites of ANP and CP. Levels of leucine, isoleucine and valine were increased in the ANP group, whereas an opposite trend was observed in the CP group. Increases in phosphocholine, glycerophosphocholine and choline levels, and decreases in fatty acids, lactate, betaine and glycine levels were observed in both the ANP and $\mathrm{CP}$ groups. Additionally, the lipid content in the CP group was higher than that observed in the ANP group. An increase in taurine levels was observed only in the $\mathrm{CP}$ group. In conclusion, pancreatitis causes a disruption of the metabolism in the pancreas at a molecular level, with increased taurine levels being a potential metabolite indicator for those with $\mathrm{CP}$.
\end{abstract}

\section{Introduction}

Pancreatitis has long been recognized as a painful and potentially fatal condition. The key histopathological features include pancreatic fibrosis, acinar atrophy, inflammation, and

Correspondence to: Dr Jian-Ping Lu, Department of Radiology, Shanghai Changhai Hospital, The Second Military Medical University, 168 Changhai Road, Shanghai 200433, P.R. China E-mail: cjr.lujianping@vip.163.com

Key words: metabolic profiles, magic angle spinning, nuclear magnetic resonance, principal component analysis distorted and blocked ducts (1-3). Pancreatitis has been classified into two subtypes, acute pancreatitis (AP) and chronic pancreatitis (CP) (4). As a severe acute pancreatitis, acute necrotizing pancreatitis (ANP) usually results from pancreatic glandular necrosis and accounts for $10-40 \%$ of the mortality rate (5-8). Chronic pancreatitis is a condition characterized by progressive and irreversible damage to both exocrine and endocrine components of the gland, eventually resulting in significant exocrine insufficiency and diabetes $(9,10)$. Patients with $\mathrm{CP}$ are also at a markedly increased risk of developing pancreatic cancer (11-13).

Despite many years of experimental and clinical investigations, both in animals and in humans, the etiology and pathogenesis of pancreatitis remains unclear (10,14-16). In different countries and regions, due to geographical factors, economic status, living habits and many other variables, both the risk factors and the incidence of pancreatitis demonstrate great variability $(10,17)$. To date, all we know for certain is that the condition results from a multiplicity of factors.

Several difficulties still exist both in the areas of research and treatment. Histological techniques offer visualization of the differences between healthy and affected individuals, but they often necessitate an invasive procedure, which leads to complications. Imaging technologies provide visual information on the focus of disease, but are less helpful in early diagnosis. Genomics locates the gene that promotes pancreatic fibrosis, but gene expression is restricted by environmental factors (18-21). The classification of pancreatitis remains complicated and imprecise $(22,23)$, and a correlation between morphological changes and pancreatic function has yet to be established (24,25). However, as the incidence of pancreatitis is increasing, it is imperative to explore its pathogenesis, so as to aid in the diagnosis and treatment, especially at the early stage of the disease.

In general, in the presence of inflammation of the pancreas, metabolite abnormalities appear before transformation of the tissue structure and changes in functions occur. Detection of abnormalities in metabolite levels facilitates a greater understanding of the pathophysiological events and aids in the early diagnosis of the disease (26). Clinically, serum amylase and serum lipase are widely used as markers of pancreatitis. However, these markers are not sensitive, show low specificity and are primarily used as additional tools (27). Abnormal levels of serum amylase and serum lipase are caused by 
a block in the excretion of pancreatic juice, which causes changes in pancreatic function. As the second largest gland in the body, the pancreas plays a critical role in the metabolism. The metabolic profile of the pancreas demonstrates abnormal characteristics when the organ is affected by certain diseases.

In the present study, we investigated whether it is possible to obtain information that could assist us in our understanding of the pathogenesis of pancreatitis and facilitate our ability to differentiate between ANP and CP at a molecular level, by analyzing pancreatic tissue samples using a metabolic profiling strategy. To clarify the metabolic characteristics of pancreatitis, disease models of ANP and CP were created experimentally, using male Wistar rats. The metabolic profile of the pancreas was investigated using high-resolution proton magic angle spinning nuclear magnetic resonance $\left({ }^{1} \mathrm{H}\right.$ MAS NMR) spectroscopy (28-30) of intact tissue, which was obtained from the animals. The processed NMR spectra of the pancreatic tissue were analyzed using principal component analysis (PCA) $(31,32)$ in order to visualize separation among the samples and to extract characteristic metabolites of ANP and $\mathrm{CP}$.

\section{Materials and methods}

Animals. A total of 22 male Wistar rats ( 2 months old, weighing 200-220 g, obtained from the experimental animal center of the Second Military Medical University, Shanghai, China) were kept under environmentally controlled conditions (temperature, $22 \pm 2^{\circ} \mathrm{C}$; light/dark cycle, $12 / 12 \mathrm{~h}$; relative humidity, 60-70\%). The rats were divided into three groups: the control group $(n=9)$, the ANP group $(n=7)$ and the CP group $(n=6)$. The disease model of $C P$ was induced via the administration of a single dose of DBTC solution (intravenous, $8 \mathrm{mg} / \mathrm{kg}$ ), which was composed of DBTC (Sigma, St. Louis, MO, USA), alcohol and glycerol. The molar ratio of DBTC, alcohol and glycerol was 1:2:3 (33). An equivalent dose of saline solution was administered to the control group, which was maintained under the same conditions as the $\mathrm{CP}$ group. The disease model of ANP was induced via two injections of an L-arginine solution $(20 \%, 2.5 \mathrm{mg} / \mathrm{g})$ with a 1-h interval (34). The study was approved by the local Animal Ethics Committee of the Shanghai Changhai Hospital Ethics Committee, Shanghai, China.

Tissue samples. Twelve hours after the injections of the L-arginine solution, the ANP group mice ( 2 months old, weighing 200-220 g) were sacrificed. Two months after injection of the DBTC/saline solution, the CP group and the control group mice ( $\sim$ months old, weighing 310-330 g) were sacrificed. The pancreatic tissues were excised from the middle sections in each group, in order to minimize variation due to possible topographical differences. Each tissue sample was cut into two parts: one was placed in formalin for histological tests; the other was transferred into cryo-tubes, labeled with unique identifiers, snap-frozen in liquid nitrogen and stored at $-80^{\circ} \mathrm{C}$ prior to ${ }^{1} \mathrm{H}$ MAS NMR analysis.

Histology. The tissue samples that had been placed in formalin (4\%) were cut, embedded in paraffin and processed routinely. Sections $(4 \mu \mathrm{m})$ for all samples were stained with
$H \& E$ and then examined under a light microscope. Two experienced pathologists reviewed all the sections. In the $\mathrm{CP}$ group, the lobular structure was found to be destroyed, the acini had disappeared, collagen fibers had proliferated and an inflammatory cell infiltrate was present. Pancreatic tissue from the ANP group revealed the presence of edema, hemorrhage and yellow-white calcification spots visible with the naked eye. Using light microscopy, it was observed that the acinus structure was destroyed, there were large areas of tissue putrescence, red blood cells appeared in the pancreatic parenchyma and many inflammatory cells had infiltrated the tissue spaces (Fig. 1). No marked pathological changes in the pancreatic tissue were observed in the control group.

MAS NMR experiments. ${ }^{1} \mathrm{H}$ MAS NMR experiments were recorded using an AVANCE II 600 spectrometer equipped with a $4-\mathrm{mm}$ double resonance $\left({ }^{1} \mathrm{H} /{ }^{13} \mathrm{C}\right)$ MAS probe $\left({ }^{1} \mathrm{H}\right.$ frequency at $600.13 \mathrm{MHz}$; Bruker Biospin, Germany). Tissue samples of $\sim 15 \mathrm{mg}$ were cut and placed into a 4-mm outer diameter zirconia MAS rotor with drops of $\mathrm{D}_{2} \mathrm{O}$ as the field lock and spun at $5 \mathrm{kHz}$. Spectra were acquired at $300.0 \mathrm{~K}$ using Carr-Purcell-Meiboom-Gill (CPMG) pulse sequence [relaxation delay (RD)- $90^{\circ}-\left(\tau-180^{\circ}-\tau\right)_{\mathrm{n}}$-acquire free induction decay (FID)] with water pre-saturation during the relaxation delay of $2 \mathrm{sec}$. CPMG pulse sequence was applied as a T2 filter to moderately attenuate signals from the macromolecules with short spin-spin relaxation time. A spin relaxation delay $(2 \mathrm{n} \tau)$ of $180 \mathrm{~ms}$ was used. The main parameters used for ${ }^{1} \mathrm{H}$ MAS NMR experiments were: spectra width $(\mathrm{SW})=$ $15 \mathrm{kHz}$; data points (TD) $=64 \mathrm{k}$ and number of scans $(\mathrm{NS})=$ 160. Spectral assignments were confirmed by 2-dimensional ${ }^{1} \mathrm{H}-{ }^{1} \mathrm{H}$ correlation spectroscopy (COSY) and ${ }^{1} \mathrm{H}-{ }^{1} \mathrm{H}$ total correlation spectroscopy (TOCSY) together with information from published data. The stability of the tissue samples was validated by repeating a 1-dimensional NMR experiment after overall acquisition. No biochemical degradation was observed in any of the tissue samples.

Principal component analysis. Spectral data were carefully manually phased and baseline-corrected using Topspin 3.1 software (Bruker Biospin). All free induction decays (FIDs) were multiplied with a $0.5-\mathrm{Hz}$ exponential line broadening prior to Fourier transformation into $128 \mathrm{~K}$ points. Each ${ }^{1} \mathrm{H}$ MAS NMR spectrum was segmented into 170 regions with equal width $(0.05 \mathrm{ppm})$ over the chemical shift region $\delta 0.50-9.00 \mathrm{ppm}$ and the signal intensity in each region was integrated using AMIX (version 3.9.5, Bruker Biospin) software. The region $\delta 4.60-5.10 \mathrm{ppm}$ was removed in order to minimize the effect of the imperfect baseline caused by the water suppression. Prior to PCA, each integral region was normalized by dividing by the sum of all the utilized integral regions for each spectrum. In order to minimize the effects of the lipids, PCA was again performed for the ${ }^{1} \mathrm{H}$ NMR spectrum over the range $\delta 2.9-9.0 \mathrm{ppm}$ with removal of the region $\delta 4.60-5.50 \mathrm{ppm}$, reducing to 122 regions of equal width (0.05 ppm). PCA was used to calculate a new set of values of uncorrelated variables from linear combinations of the observations of possibly correlated variables within the data. These new uncorrelated variables are called principal components, and the distribution of their values (scores) permits the simple 


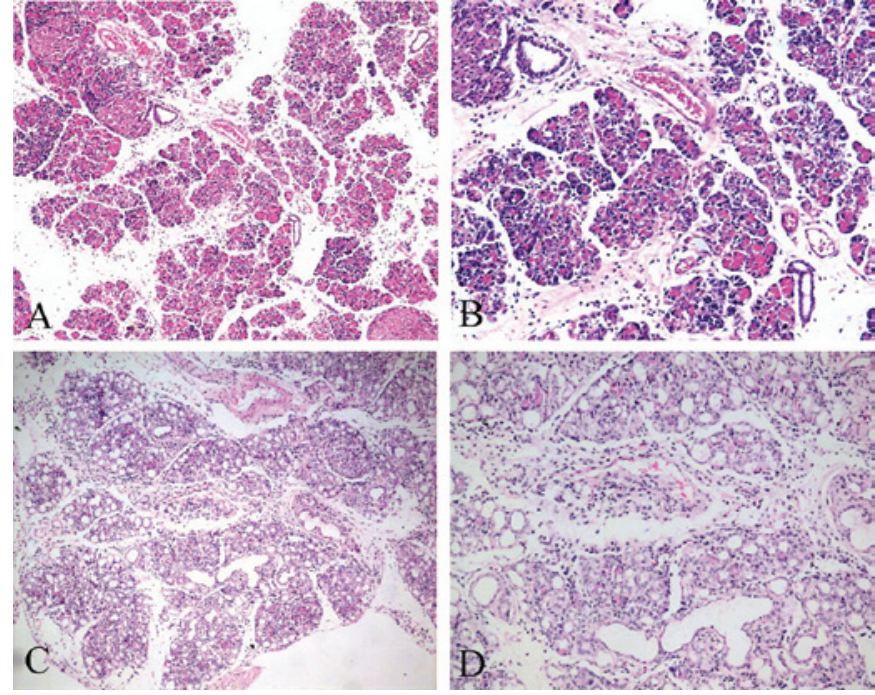

Figure 1. (A and B) Representative photomicrographs of acute necrotizing pancreatitis. (A) H\&E, magnification, x100. (B) H\&E, magnification, $x 400$ (C and D) Representative photomicrographs of chronic pancreatitis. (C) $\mathrm{H} \& \mathrm{E}$, magnification, $\mathrm{x} 100$. (D) H\&E, magnification, $\mathrm{x} 400$.
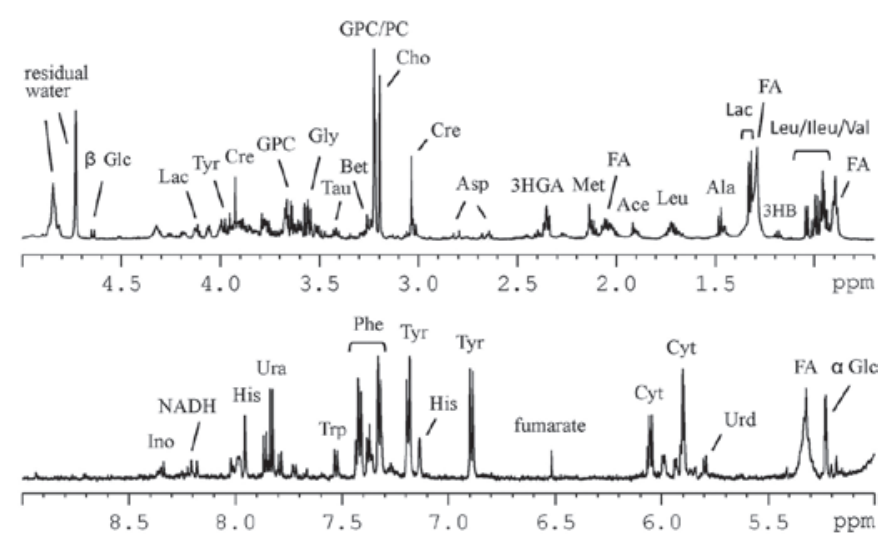

Figure 2. Assignment of proton magic angle spinning nuclear magnetic resonance spectra to pancreatic tissue with ANP. The region of $\delta 5.00-$ $9.00 \mathrm{ppm}$ is zoomed-in 16 times of that in $80.70-5.00 \mathrm{ppm}(600 \mathrm{MHz}, \mathrm{CPMG}$ pulse sequence, temperature $300.0 \mathrm{~K}$ ). 3HB, 3-hydroxybutyrate; 3HGA, 3 -hydroxyglutaric acid; $\alpha$-Glc, $\alpha$ glucose; $\beta$-Glc , $\beta$ glucose; Ace, acetate; Ala, alanine; Asp, aspartate; Bet, betaine; Cho, choline; Cre, creatine; Cyt, cytidine; FA, fatty acid; Glc, glucose; Gly, glycine; GPC, glycerophosphocholine; His, histidine; Ileu, iso-leucine; Ino, inosine; Lac, lactate; Leu, leucine; Met, methionine; PC, phosphocholine; Phe, phenylalanine; Tau, taurine; Trp, tryptophan; Tyr, tyrosine; Ura, uracil; Urd, uridine; Val, valine.

visualization of separation or clustering among different samples. The weightings (loadings) that are given to each integral region in calculating the principal components allows for the identification of those spectral regions for the maximum impact to the separation or clustering. Thus, we may obtain information on the biomarkers of ANP and CP by analyzing MAS NMR spectra with PCA.

\section{Results}

NMR experiments. Combining 2D NMR experimental results of the present study with published data of related literature $(31,35,36)$, we identified the main resonances in ${ }^{1} \mathrm{H}$ NMR spectra of pancreatic tissue (Fig. 2). The components, which

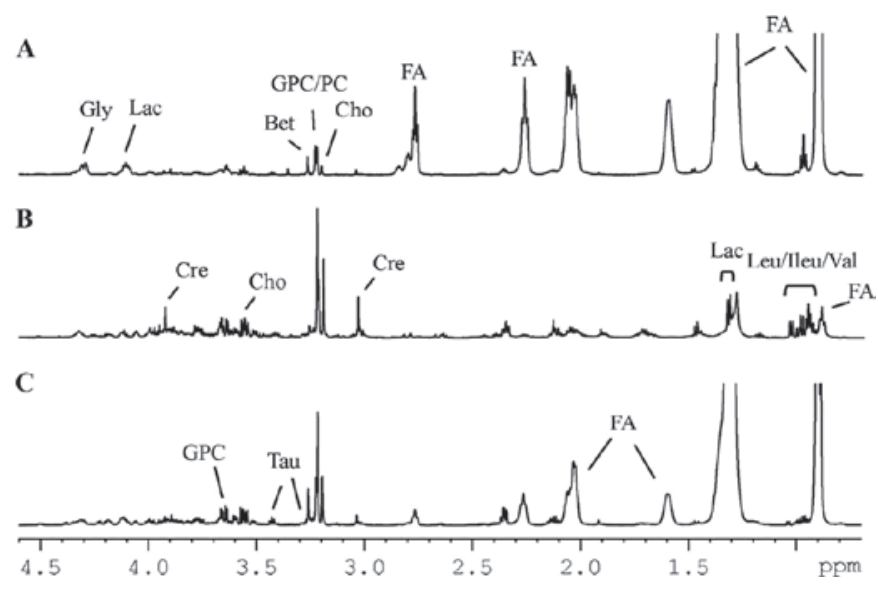

Figure 3. Three typical 600-MHz ${ }^{1} \mathrm{H}$ MAS NMR CPMG spectra obtained from a rat pancreas; (A) control, (B) acute necrotizing pancreatitis and (C) chronic pancreatitis group.
A

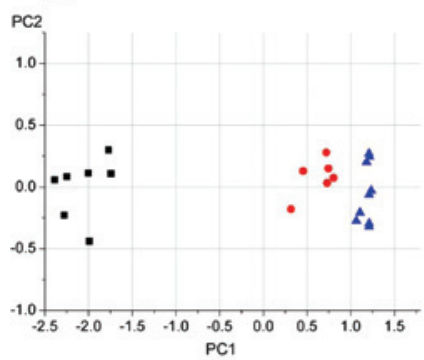

$\mathrm{C}$

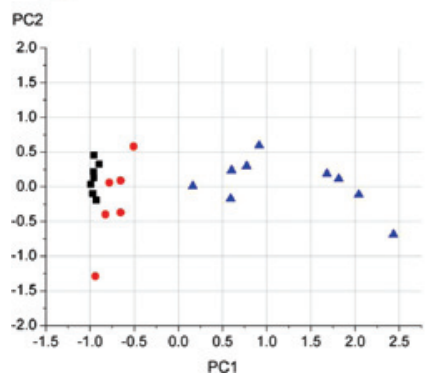

B

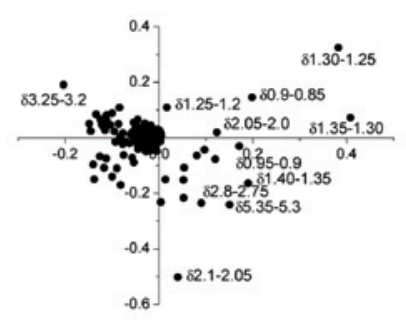

$\mathrm{D}$

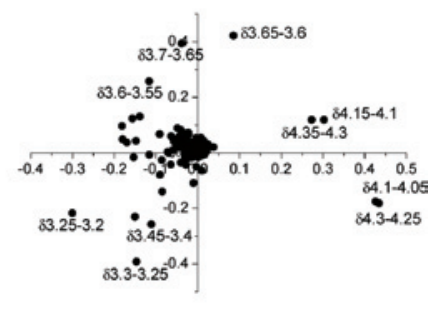

Figure 4. (A and C) Principal component analysis scores plots and (B and D) loadings plots. (A and B) From data of the ${ }^{1} \mathrm{H}$ CPMG NMR spectra (80.50-9.00 ppm). (C and D) From data of the ${ }^{1} \mathrm{H}$ CPMG NMR spectra ( $\delta 2.9-9.0 \mathrm{ppm})$. $\triangle$ Control group; macute necrotizing pancreatitis group; -chronic pancreatitis group.

include leucine (Leu), iso-leucine (Ileu), valine (Val), fatty acids (FA), lactate (Lac), alanine (Ala), leucine (Leu), acetate (Ace), methionine (Met), 3-hydroxyglutaric acid (3HGA), aspartate (Asp), creatine (Cre), choline (Cho), phosphocholine (PC), glycerophosphocholine (GPC), betaine (Bet), taurine (Tau), glycine (Gly), tyrosine (Tyr), $\beta$ glucose $(\beta-G l c), \alpha$ glucose $(\alpha-\mathrm{Glc})$, uridine (Urd), cytidine (Cyt), tyrosine (Tyr), histidine (His), phenylalanine (Phe), tryptophan (Trp), uracil (Ura) and inosine (Ino), and the locations, are clearly demonstrated.

Fig. 3 shows representative spectra for ${ }^{1} \mathrm{H}$ MAS NMR of the control group, the ANP group and the $\mathrm{CP}$ group. Compared to Fig. 3A, some peaks in Fig. 3B and C are similar; for example, an increase in GPC/PC and Cho, and a decrease in FA, Lac, Bet and Gly were observed in both the CP and ANP groups, 
despite a more marked fluctuation in the CP group. However, the other peaks revealed a distinctively opposite trend, with Leu, Ileu and Val decreasing in the CP group, but increasing in the ANP group. Tau was only increased in the CP group. In addition, the $\mathrm{CP}$ group showed higher lipid levels than the ANP group.

Principal component analysis. In the scores plot obtained from the CPMG NMR spectra ( $80.50-9.00 \mathrm{ppm})$ (Fig. 4A), each group demonstrated its own individual within-group consistency. The CP group was closer to the control group and further from the ANP group in PC1. The corresponding loadings plot (Fig. 4B) revealed that lipids ( $\delta 0.95-0.85, \delta 1.40-1.25$, $\delta 2.10-2.00$ and $\delta 5.35-5.30 \mathrm{ppm})$, Lac ( $\delta 1.40-1.35 \mathrm{ppm})$ and GPC/PC ( $83.25-3.2 \mathrm{ppm})$ contributed the most to the separation of the three groups in the scores plot (Fig. 4A). In order to decrease the effects of the lipid metabolites, PCA was applied to the CPMG NMR spectra range ( $82.9-9.00 \mathrm{ppm})$ with minimizing baseline effects of imperfect water saturation to obtain scores and a loadings plot (Fig. 4C and D). In Fig. 4C, all three groups were clearly separated, with each group demonstrating its own individual within-group similarities. The CP group was closer to the ANP group and further away from the control group in $\mathrm{PC} 1$, which is different from the results of Fig. 4A. The corresponding loadings plot (Fig. 4D) revealed that Gly ( $\delta 4.35-4.25 \mathrm{ppm})$, Lac ( $\delta 4.15-4.05 \mathrm{ppm})$, Cho

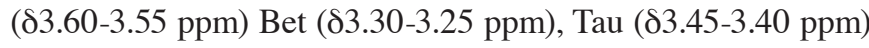
and GPC/PC ( $83.75-3.65$ and $\delta 3.25-3.2 \mathrm{ppm})$ contributed the most to the separations observed in Fig. 4C.

\section{Discussion}

In our study, Gly, Lac, Bet, Cho, GPC/PC, Leu/Ileu/Val and various lipids, all correlated with ANP and CP. ANP demonstrated higher Leu/Ileu/Val and lower Tau and FA levels when compared to $\mathrm{CP}$. These findings demonstrate that pancreatitis causes a clear disruption of the pancreatic metabolism at a molecular level, and suggest that Leu/Ileu/Val, lipids and Tau are all potential metabolic markers for the differentiation of ANP from CP.

With in vivo ${ }^{1} \mathrm{H}$ MRS technology, clinical research has concluded that lipid in chronic focal pancreatitis is significantly less than that observed in pancreatic carcinoma tissues (37). Cancerous tissues generally have high lipid content, but the reverse is not always true for other pancreatic diseases, as tissue lipid content varies widely due to factors such as genetics, aging, gender and the environment. From the ${ }^{1} \mathrm{H}$ MAS NMR spectra (Fig. 3) we observed that lipid levels, when compared to those of the control group, were slightly decreased in the CP group, whereas they were markedly decreased in the ANP group. The different weighting of lipids brought out the separation of the three groups in the scores plot (Fig. 4A), which could also be certified by the results of the loadings plot (Fig. 4B). This result suggests that the $\mathrm{CP}$ group has a more active metabolism than the ANP group. The difference in lipid levels between the CP and the ANP groups showed the characteristic metabolomics that appear as a result of the inflammatory process within the pancreas.

From MRS studies on tissues in vivo, the choline signal is primarily observed as a prominent singlet at $3.2 \mathrm{ppm}$ that includes contributions from free choline, GPC and PC, and is often referred to as 'total choline' (38-40). In the present study, we obtained high resolution signals of Cho, PC and GPC at the chemical shifts 3.19, 3.21 and $3.22 \mathrm{ppm}$, respectively. The ${ }^{1} \mathrm{H}$ MAS NMR results showed that Cho, PC and GPC were increased in both the ANP and CP groups. The total choline metabolites have been chosen as biomarkers in various carcinoma studies, as they tend to increase as a result of increased membrane concentrations during enhanced proliferation of cancer cells $(41,42)$. As the modest MAS rates $(5 \mathrm{kHz})$ are less than the dipole-dipole interactions of the cell membrane, it is unlikely that membrane-bound Cho, PC and GPC are easily demonstrated $(31,43)$. Hence, Cho, PC and GPC detected in our study were mainly derived from free molecules within the pancreas. The increased Cho, PC and GPC in ANP and $\mathrm{CP}$ may result from the blockage of Cho-kinase and $\mathrm{PC}$ transferase, or from the consumption of PC through the CDP-Cho pathway. Thus, one may deduce that increased total choline represents a characteristic metabolite in the process of both $\mathrm{CP}$ and ANP.

In order to lessen the effects of lipids and to explore the low-molecular-weight metabolites that are relevant to the pathophysiological processes, the regions of 2.9-9.00 ppm in the NMR spectra were analyzed by PCA (Fig. 4C and D). Compared to Fig. 4A, it can be seen that the $\mathrm{CP}$ group has migrated towards the ANP group in PC1 (Fig. 4C), which is due to their similar inherent metabolic profiles, apart from a difference in lipid content, and the chemical shift of relevant metabolites could be obtained from the loadings plot (Fig. 4D). The elevation observed in Tau played an important role in the discrimination of ANP from CP. Tau is a free intracellular amino acid that is reported to have a number of biological functions, including osmoregulation and modulation of neurotransmitter action. It is chiefly obtained from food, although it may also be synthesized from other sulfur-containing amino acids $(38,44)$. The metabolic actions of Tau are correlated with the control of serum cholesterol levels, blood sugar and cellular calcium levels (31). Thus, in the process of CP and ANP, normal Tau levels serve to maintain normal serum cholesterol levels, blood sugar and cellular calcium levels. To maintain the serum amylase levels, blood sugar levels are or approach normal levels, and an increase in Tau levels is necessary. Tissue putrescence appears in ANP, and therefore the ability to obtain Tau from food may be restricted, and Tau levels may decrease sharply in ANP.

From Figs. 3 and 4D, we conclude that the decrease in Gly, Lac and Bet in the CP and ANP groups caused the distinct separation in the scores plot (Fig. 4C). Gly is a simple amino acid that acts as an inhibitory neurotransmitter and antioxidant (45). Lac, the product of anaerobic glycolysis, is increased in the presence of hypoxia and ischemia (38). Our results showed that Gly and Lac were markedly decreased in both the ANP and the CP groups, due to their association with a metastatic potential, as has been reported by Sitter et al (46). Compared to other low-molecular weight-metabolites, Gly and Lac demonstrated the most noticeable differences between all three groups. Bet donates methyl groups for remethylation of homocysteine to methionine and dimethylglycine, which supports normal liver and pancreatic function, cellular replication and detoxification reactions (31). Therefore, the depletion of Bet metabolites in CP and ANP for the detoxification of the 
pancreas is necessary and it is possible that the levels of Bet decrease both in CP and ANP.

Although the number of samples in our study was limited, the potential of ${ }^{1} \mathrm{H}$ MAS NMR for the investigation of physiological and pathological consequences of pancreatic diseases should not be ignored. The above results clearly demonstrate that the metabolic profiles and characteristics of ANP and CP can be discriminated characteristically without the disturbance of various lipid contents by ${ }^{1} \mathrm{H}$ MAS NMR coupled with PCA. As metabolite changes observed by ${ }^{1} \mathrm{H}$ MAS NMR always occur before morphological changes investigated by imaging technologies, the findings will clearly be of benefit to pathological research, early diagnosis and therapeutic evaluation of pancreatic diseases.

\section{Acknowledgements}

This study was supported by the National Natural Science Foundation of China (No. 30870709).

\section{References}

1. Spanier BW, Dijkgraaf MG and Bruno MJ: Trends and forecasts of hospital admissions for acute and chronic pancreatitis in the Netherlands. Eur J Gasroenterol Hepatol 20: 653-658, 2008.

2. Mitchell RM, Byrne MF and Baillie J: Pancreatitis. Lancet 361: 1447-1455, 2003.

3. Braganza JM: Evolution of pancreatitis. In: The Pathogenesis of Pancreatitis. Braganza JM (ed.). Manchester University Press, Manchester, 1991.

4. Sarles H: Pancreatitis: Symposium of Marseille, 1963. Karger, Basel, 1965.

5. Varon TH and Morgan DE: Acute necrotizing pancreatitis. N Engl J Med 340: 1412-1417, 1999.

6. Bai Y, Liu Y, Jia L, et al: Severe acute pancreatitis in China: etiology and mortality in 1976 patients. Pancreas 35: 232-237, 2007.

7. Felderbauer P, Muller C, Bulut K, et al: Pathophysiology and treatment of acute pancreatitis: new therapeutic targets - a ray of hope? Basic Clin Pharmacol Toxicol 97: 342-350, 2005.

8. Raraty MG, Connor S, Criddle DN, Sutton R and Neoptolemos JP Acute pancreatitis and organ failure: pathophysiology, natural history, and management strategies. Curr Gastroenterol Rep 6: 99-103, 2004.

9. Etemad B and Whitcomb DC: Chronic pancreatitis: diagnosis, classification, and new genetic developments. Gastroenterology 120: 682-707, 2001

10. Witt H, Apte MV, Keim V and Wilson JS: Chronic pancreatitis: challenges and advances in pathogenesis, genetics, diagnosis, and therapy. Gastroenterology 132: 1557-1573, 2007.

11. Lowenfels AB, Maisonneuve P and Whitcomb DC: Risk factors for cancer in hereditary pancreatitis. International Hereditary Pancreatitis Study Group. Med Clin North Am 84: 565-573, 2000.

12. Talamini G, Falconi M, Bassi C, et al: Incidence of cancer in the course of chronic pancreatitis. Am J Gastroenterol 94: 1253-1260, 1999.

13. Lowenfels AB, Maisonneuve P, Cavallini G, et al: Pancreatitis and the risk of pancreatic cancer. N Engl J Med 328: 1433-1437, 1993.

14. Wu BU: Prognosis in acute pancreatitis. CMAJ 183: 673-677, 2011

15. Kamisawa T: Is it time to reconsider autoimmune pancreatitis? J Gastroenterol 41: 1240-1241, 2006.

16. Tsai MJ, Chen C, Chen SH, Huang YT and Chiu TH: Pomalidomide suppresses cerulein-induced acute pancreatitis in mice. J Gastroenterol 46: 822-833, 2011.

17. Strate L, Knoefel W, Yekebas E and Izbicki JR: Chronic pancreatitis: etiology, pathogenesis, diagnosis and treatment. Int J Colorectal Dis 18: 97-106, 2003.

18. Al-Haddad M and Eloubeidi MA: Diagnostic and therapeutic applications of endoscopic ultrasound-guided punctures. Dig Dis 26: 390-397, 2008.
19. DiMagno E, Layer $\mathrm{P}$ and Clain J: Chronic pancreatitis. In: The Pancreas: Biology, Pathophysiology and Disease. Raven, New York, 1993

20. Bolog N, Constantinescu G, Oancea I, et al: Magnetic resonance imaging of bile and pancreatic ducts: a retrospective study. Rom J Gastroenterol 13: 91-97, 2004.

21. Cavestro GM, Zuppardo RA, Bertolini S, et al: Connections between genetics and clinical data: role of MCP-1, CFTR, and SPINK-1 in the setting of acute, acute recurrent, and chronic pancreatitis. Am J Gastroenterol 105: 199-206, 2010.

22. Clain JE and Pearson RK: Diagnosis of chronic pancreatitis: is a gold standard necessary? Surg Clin North Am 79: 829-845, 1999.

23. Büchler MW, Martigone ME, Friess H and Malfertheiner P: A proposal for a new clinical classification of chronic pancreatitis. BMC Gastroenterol 9: 93-101, 2009.

24. Maartense S, Ledeboer M and Masclee AAM: Chronic pancreatitis: relation between function and morphology. Dig Liver Dis 36: 61-67, 2004.

25. Cohn JA: Reduced CFTR function and the pathobiology of idiopathic pancreatitis. J Clin Gastroenterol 39: S70-S77, 2005.

26. Wang J, Ma C, Liao Z, Tian B and Lu JP: Study on chronic pancreatitis and pancreatic cancer using MRS and pancreatic juice samples. World J Gastroenterol 17: 2126-2130, 2011.

27. Lesi C, Melzi D'Eril GV, Pavesi F, et al: Clinical significance of serum pancreatic enzymes in the quiescent phase of chronic pancreatitis. Clin Biochem 18: 235-238, 1985.

28. Beckonert O, Keun HC, Ebbels TM, Bundy J, Holmes E, Lindon JC and Nicholson JK: Metabolic profiling, metabolomic and metabonomic procedures for NMR spectroscopy of urine, plasma, serum and tissue extracts. Nat Protoc 2: 2692-2703, 2007.

29. Moestue S, Sitter B, Bathen TF, Tessem MB and Gibbestad IS: High-resolution magic angle spinning (HR MAS) MR spectroscopy in metabolic characterization of human cancer. Prog Nucl Magn Reson Spectrosc 54: 239-254, 2009.

30. Lindon JC, Beckonert OP, Holmes E and Nicholson JK: Highresolution magic angle spinning NMR spectroscopy: application to biomedical studies. Prog Nucl Magn Reson Spectrosc 55: 79-100, 2009

31. Fang F, He XH, Deng HW, Chen Q, Lu JP, Spraul M and Yu YH: Discrimination of metabolic profiles of pancreatic cancer from chronic pancreatitis by high-resolution magic angle spinning $1 \mathrm{H}$ nuclear magnetic resonance and principal components analysis. Cancer Sci 98: 1678-1682, 2007.

32. Holmes E, Nicholls AW, Lindon JC, et al: Chemometric models for toxicity classification based on NMR spectra of biofluids. Chem Res Toxicol 13: 471-478, 2000.

33. Merkord J, Weber H, Sparmann G, Jonas L and Hennighausen G: The course of pancreatic fibrosis induced by dibutyltin dichloride (DBTC). Ann NY Acad Sci 880: 231-237, 1999.

34. Dabrowska A, Jacewicz D, Lapinska A, et al: Pivotal participation of nitrogen dioxide in 1-arginine induced acute necrotizing pancreatitis: protective role of superoxide scavenger 4-OH-TEMPO. Biochem Biophys Res Commun 326: 313-320, 2005.

35. Griffin JL, Mannb CJ, Scottc J, Shoulders CC and Nicholoson JK: Choline containing metabolites during cell transfection: an insight into magnetic resonance spectroscopy detectable changes. FEBS Lett 509: 263-266, 2001.

36. Garrod S, Humpfer E, Spraul M, et al: High-resolution magic angle spinning $1 \mathrm{H}$ NMR spectroscopic studies on intact rat renal cortex and medulla. Magn Reson Med 41: 1108-1118, 1999.

37. Cho SG, Lee DH, Lee KY, Ji H, Lee KH, Ros PR and Sun CH: Differentiation of chronic focal pancreatitis from pancreatic carcinoma by in vivo proton magnetic resonance spectroscopy. J Comput Assist Tomogr 29: 163-169, 2005.

38. Govindaraju V, Young K and Maudsley AA: Proton NMR chemical shifts and coupling constants for brain metabolites. NMR Biomed 13: 129-153, 2000.

39. Miller BL, Chang L, Booth R, et al: In vivo $1 \mathrm{H}$ MRS choline: correlation within vitro chemistry/histology. Life Sci 58: 1929-1935, 1996.

40. Tan J, Bluml S, Hoang T, Dubowitz D, Mevenkamp G and Ross B: Lack of effect of oral choline supplement on the concentrations of choline metabolites in human brain. Magn Reson Med 39: S1005-S1010, 1998.

41. Cheng LL, Anthony DC, Comite AR, Black PM, Tzika AA and Gonzalez RG: Quantification of microheterogeneity in glioblastoma multiforme with ex vivo high-resolution magic-angle spinning (HRMAS) proton magnetic resonance spectroscopy. Neuro Oncol 2: 87-95, 2000. 
42. Loening NM, Chamberlin AM, Zepeda AG, Gonzalez RG and Cheng LL: Quantification of phosphocholine and glycerophosphocholine with 31P edited 1H NMR spectroscopy. NMR Biomed 18: 413-420, 2005.

43. Barker PB, Breiter SN, Soher BJ, et al: Quantitative proton spectroscopy of canine brain: in vivo and in vitro correlations. Magn Reson Med 32: 157-163, 1994.

44. Hardy DL and Norwood TJ: Spectral editing techniques for the in vitro and in vivo detection of taurine. J Magn Reson 133: 70-78, 1998.
45. Kinoshita Y, Kajiwara H, Yokota A and Koga Y: Proton magnetic resonance spectroscopy of brain tumors: an in vitro study. Neurosurgery 35: 606-613, 1994.

46. Sitter B, Bathen TF, Hagen B and Gibbestad IS: Cervical cancer tissue characterized by high resolution magic angle spinning MR spectroscopy. MAGMA 16: 174-181, 2004. 